\title{
Performance Analysis of A CDMA ALOHA Network with Channel-sensing
}

\author{
F. L. Lo \\ fllo@hkueee.hku.hk \\ T. S. Ng \\ tsng@hkueee.hku.hk \\ T. I. Yuk \\ tiyuk@hkueee.hku.hk
Department of Electrical and Electronic Engineering
The University of Hong Kong \\ Pokfulam Road, Hong Kong \\ Tel.: (852) 28578406 \\ Fax.: (852) 25598738
}

\begin{abstract}
It has been shown in [1] that the throughput-delay performance of a CDMA ALOHA network, where each station is assigned a spreading code so that each effectively has its own 'virtual channel', and where channel sensing and collision detection are also used, is much better than that of a simple CSMA-CD network. Such a system, however, is difficult to implement. A simpler system that may provide good performance and that is easy to implement is a CDMA ALOHA network with just channel sensing. The channel sensing can be provided by a simple correlator and threshold device. In this paper, the performance of such a network is investigated, and some comparisons are made with those of the simple CSMA-CD network and the CDMA ALOHA network with both channel sensing and collision detection. It is shown that when the CDMA-CS network is stable, its performance is not much worse than that of a CDMA-CS/CD network, and much better than that of a simple single channel CSMA-CD system.
\end{abstract}

\section{INTRODUCTION}

It has been shown in [1] that a full-duplex CDMA ALOHA network with channel sensing and collision detection (which we shall call a CDMA$\mathrm{CS} / \mathrm{CD}$ network) has a much better throughput-delay performance compared to a simple CSMA-CD network. The implementation of a CDMA-CS/CD network, however, is not simple, as either a separate receiver needs to be included in each station to be used for both channel sensing and detection of packet collisions, or each time slot needs to be divided into two, with a longer portion for information transmission, and a shorter portion for handshaking. If the former method is employed, because of the near-far problem [2], the collision detection receiver must be isolated from the station's transmitter so that it wouldn't be overwhelmed by the station's own strong signal. If the latter approach is adopted, with the first packet transmitted acting as a reservation packet, somewhat similar to the halfduplex network studied in [3], then a portion of each slot must be freed from information transmission in order for handshaking to be done without interference. This is an overhead that lowers the efficiency of the network.

A solution that readily comes to mind is to use channel sensing only and not collision detection in the CDMA network. Its performance will not match that of a CDMA-CS/CD network as some collisions will occur and some slots will be wasted, but its simplicity and its improvement in performance over a simple CSMA-CD network may make such networks practical. In this paper, we shall evaluate the performance of such a CDMA-CS network and show that its performance, though not as good as that of a CDMA-CS/CD network, is much better than that of a simple CSMA-CD system.

In Section II, we shall describe the model for the full-duplex CDMA-CS network and discuss how it can be implemented. This model is analyzed using Equilibrium Point Analysis (EPA) in Section III. Some numerical results are presented in Section IV, and comparisons of the performance of the CDMA-CS network with those of the CDMA$\mathrm{CS} / \mathrm{CD}$ and CSMA-CD networks are also given. The paper ends with Section $\mathrm{V}$ where some conclusions are provided.

\section{NETWORK MODEL}

Time is divided into slots, each of duration $\tau$, which is long enough for transmission from any station to reach all other stations in the network. Each station is assigned a specific spreading code, as in the "receiver-based code" scheme of [4], or a specific phase of the same spreading code if the system is synchronized, as in the CPA networks of [5], with which it will receive messages.

Stations waiting to be presented with new messages are called idle and they are presented with newly generated messages at the rate of $s$ per slot, where $s<1$ and can therefore be treated as the message generation probability per slot for each station. Once a new message arrives at an idle station, it will activate its sensing receiver to sense the channel of the intended recipient $k$. This sensing receiver can be made up of just a correlator and a threshold device as all it needs to do is load the 
correlator with the spreading code of the intended receiver and determine if the energy output of the correlator at the carrier frequency exceeds a certain threshold. If it does, the station will assume that some other station is transmitting down channel $k$ and enter the blocked mode.

Should the station sense that the channel of the intended receiver is free, it will start transmitting the message with probability one during the next slot. If no other stations start transmission during the same slot down channel $k$, then the station is said to have captured the channel. Should one or more other stations attempt to transmit during that slot down channel $k$, then a collision of messages will occur and the channel is said to be wasted. Messages are made up of a random number of packets, each taking $\tau$ to send, which is geometrically distributed with an average of $l$. The captured or wasted channels, which we shall call "busy", will thus remain in their state on average for $l$ slots. Stations are assumed to know immediately at the end of the transmissions whether the transmissions are successful. This is obviously an approximation as in practical systems, stations will only know whether they are successful when they receive acknowledgment packets. In a lightly loaded network, however, this approximation is good since recipients can acknowledge messages almost immediately.

Blocked stations will carry on sensing the channel of the intended receiver until it becomes free, and then attempt to retransmit with probability $p$ during each subsequent slot. Stations which are unsuccessful in their transmissions because of message collisions also enter the blocked mode when they finish their unsuccessful transmissions, and they too will attempt to retransmit with probability $p$ each slot after sensing that the channel of the intended receiver is free. Stations are assumed to have only one buffer to hold messages, so blocked stations will refuse any new messages until they become idle again.

For a very lightly loaded network, even the simple sensing receiver may not be needed, and stations may use their receivers for both channei sensing and information reception. The only inaccuracies caused by this to the analysis to be presented are: i) stations already receiving messages have to defer transmissions till the reception is complete, and ii) some messages may not be received correctly because intended receivers may be sensing some other channels. These occurrences will be rare in a lightly loaded network, and the effects on average behavior caused by such aberrations should be negligible. The behavior of such a network will, however, not be covered in this paper. In the rest of the paper, all stations are assumed to have a separate channel sensing receiver.
The system is also assumed to be ideal and all imperfections such as timing jitter, multi-path fading, finite cross-correlation between signals meant for different stations and noise, are ignored. Messages that do not collide are assumed to be received correctly.

\section{ANALYSIS OF MODEL}

Equilibrium Point Analysis (EPA) makes use of the fact that the system in the long run will operate near the point in system phase space where the message input balances the message output. The equilibrium point yields the equilibrium message throughput and number of blocked stations, which can then be treated as the average of these values. It was shown in [6], where EPA was first used for a slotted ALOHA system, and where it was called "fluid-flow approximation" that such an approximation is good provided that the system has a large number of stations and that the system has only one equilibrium point.

Let us consider a CDMA channel-sensing network with $N$ stations. First we wish to determine if a system will be stable and uncongested, as defined in [6]. Suppose the system has $n_{0}$ idle stations. For a busy channel $k$ with $n_{k}$ blocked stations, the increase in $n_{k}$ is just the average of newly generated messages meant for receiver $k$. This is

$$
\begin{aligned}
& \Delta n_{k} \text { (busy) }=n_{0} \frac{s}{N}\left(1-\frac{s}{N}\right)^{n_{0}-1} \\
& +2\left(\begin{array}{l}
n_{0} \\
2
\end{array}\right)\left(\frac{s}{N}\right)^{2}\left(1-\frac{s}{N}\right)^{n_{0}-2}+\cdots+n_{0}\left(\frac{s}{N}\right)^{n_{0}}
\end{aligned}
$$

For a free channel $k$ with $n_{k}$ blocked stations, the increase in blocked stations is

$$
\begin{aligned}
& \Delta n_{k}(\text { free+ })=n_{0} \frac{s}{N}\left(1-\frac{s}{N}\right)^{n_{0}-1}\left(1-(1-p)^{n_{k}}\right) \\
& +2\left(\begin{array}{c}
n_{0} \\
2
\end{array}\right)\left(\frac{s}{N}\right)^{2}\left(1-\frac{s}{N}\right)^{n_{0}-2}+\cdots+n_{0}\left(\frac{s}{N}\right)^{n_{0}}
\end{aligned}
$$

The decrease in $n_{k}$ for the free channel $k$ is just the probability of a blocked station capturing the channel, which is

$$
\Delta n_{k}(\text { free- })=n_{k} p(1-p)^{n_{k}-1}\left(1-\frac{s}{N}\right)^{n_{0}}
$$

A busy channel will remain captured or wasted on average for $l+1$ slots, $l$ slots to finish transmission, whether successful or not, and one more for all the stations to realize that the channel is free. A free channel will stay free for $l_{f}=1 / p_{f}$ slots before becoming busy, where $p_{f}$ is the 
probability of the free channel being wasted or captured.

$$
p_{f}=1-\left(1-\frac{s}{N}\right)^{n_{0}}(1-p)^{n_{k}}
$$

The average increase in the number of blocked stations at channel $k$ is therefore

$$
\begin{aligned}
& \Delta n_{k}(\text { average })=\frac{l+1}{l+1+l_{f}}\left[\Delta n_{k}(\text { busy })\right] \\
& +\frac{l_{f}}{l+1+l_{f}}\left[\Delta n_{k}(\text { free }+)-\Delta n_{k}(\text { free- })\right]
\end{aligned}
$$

For a system to be at equilibrium, the average message input rate $S_{\text {in }}=n_{0} s$ must be equal to the message completion rate $S_{\text {out }}=n_{t} /(l+1)$ where $n_{t}$ is the number of transmitting stations at equilibrium. Thus at equilibrium, the following equation must be satisfied:

$$
\left(N-n_{b}-n_{c}-n_{t}\right) s=\frac{n_{t}}{l+1}
$$

where $n_{b}$ is the number of blocked stations and $n_{c}$ is the number of colliding stations. If it can be shown that for all $n_{k}, 0<n_{k}<n_{b}$, where eqn. 6 is satisfied , $\Delta n_{k}$ is negative, then the system is unconditionally stable and uncongested, as any channels that become occupied, i.e., have blocked stations trying to transmit down them, will tend to clear themselves of those blocked stations. Since $n_{c}$ is not easily obtainable, we shall consider the worst case, i.e., where $n_{b}=N-n_{0}$ is maximum, and $n_{c}$ is zero. Thus we check the stability of the system by considering $\Delta n_{k}$ along the curve

$$
\left(N-n_{b}-n_{t}\right) s=\frac{n_{t}}{l+1} .
$$

Once we have determined that a system is stable and uncongested, we want to find out the equilibrium throughput and delay. To do this, we need to find out where along the curve described by eqn. 7 the system will settle down at. First, we set down all the relationships between $n_{0}, n_{c}, n_{t}$ and $n_{b}$, the number of stations idle, colliding, transmitting and being blocked, respectively. We start with the channel capture rate.

The channel capture rate of an occupied channel depends on how many blocked stations it has. Since stable uncongested systems have occupied channels that tend to clear themselves, we shall assume that all occupied channels at equilibrium have only one blocked station. The good agreement between the numbers obtained from simulation and EPA in Section IV will justify this assumption.
The capture rate when a channel is occupied by just one blocked station is

$$
\begin{aligned}
S_{c a p}(o c c) & =n_{0} \frac{s}{N}\left(1-\frac{s}{N}\right)^{n_{0}-1}(1-p) \\
& +\left(1-\frac{s}{N}\right)^{n_{0}} p
\end{aligned}
$$

The capture rate when a channel is unoccupied is

$$
S_{\text {cap }}(\text { unocc })=n_{0} \frac{s}{N}\left(1-\frac{s}{N}\right)^{n_{0}-1}
$$

The channel wastage rate for occupied channels is

$$
\begin{aligned}
S_{\text {waste }}(o c c)= & (1-p) \cdot\left[1-n_{0} \frac{s}{N}\left(1-\frac{s}{N}\right)^{n_{0}-1}\right. \\
& \left.-\left(1-\frac{s}{N}\right)^{n_{0}}\right]+p \cdot\left[1-\left(1-\frac{s}{N}\right)^{n_{0}}\right]
\end{aligned}
$$

The channel wastage rate for unoccupied channels is

$$
S_{\text {waste }}(\text { unocc })=1-n_{0} \frac{s}{N}\left(1-\frac{s}{N}\right)^{n_{0}-1}-\left(1-\frac{s}{N}\right)^{n_{0}}
$$

On average, an occupied channel will stay free for

$$
l_{c}=\frac{1}{S_{c a p}(o c c)+S_{\text {waste }}(o c c)} \text { slots }
$$

and on average, an unoccupied channel will stay free for

$$
l_{u}=\frac{1}{S_{c a p}(u n o c c)+S_{\text {waste }}(\text { unocc })} \text { slots. }
$$

When a channel is busy, it cannot be captured. Since we assumed that there are $n_{b}$ occupied channels with one blocked station each and $N-n_{b}$ unoccupied channels, the average channel capture rate for the system is therefore

$$
\begin{aligned}
& S_{\text {cap }}(\text { overage })=n_{b} \frac{l_{c}}{l_{c}+l+1} S_{c a p}(\text { occ }) \\
& \quad+\left(N-n_{b}\right) \frac{l_{u}}{l_{u}+l+1} S_{c a p}(\text { unocc }) \\
& =n_{b} f_{c} S_{c a p}(\text { occ })+\left(N-n_{b}\right) f_{u} S_{c a p}(\text { unocc })
\end{aligned}
$$

where $f_{c}$ is the fraction of time an occupied channel is free and $f_{u}$, the fraction of time an unoccupied channel is free.

At equilibrium, the capture rate of channels is equal to the message input rate since all messages being transmitted down a captured channel will be successful. Hence

$$
n_{0} s=n_{b} f_{c} S_{c a p}(o c c)+\left(N-n_{b}\right) f_{u^{u}} S_{c a p}(\text { unocc })
$$




$$
n_{b}=\frac{n_{0} s-N f_{u} S_{c a p}(\text { unocc })}{f_{c} S_{c a p}(o c c)-f_{u} S_{c a p}(\text { unocc })}
$$

Also the number of successful message completion is equal to the number of input messages

$$
\frac{n_{t}}{l+1}=n_{0} s
$$
channels is

At equilibrium, the average wastage rate of

$$
\begin{aligned}
S_{\text {waste }} & =n_{b} f_{c} S_{\text {waste }}(o c c) \\
& +\left(N-n_{b}\right) f_{u} S_{\text {waste }} \text { (unocc) }
\end{aligned}
$$

For a stable, uncongested system, $s<<1$, and therefore collisions will usually occur between only two stations, and not more, as each extra station involved in a collision lowers the probability of that collision by roughly a factor of $s / N$. Thus we shall assume that the average increase in the number of colliding stations at equilibrium for stable, uncongested systems is $2 S_{\text {waste }}$. This must be equal to the average decrease in colliding stations, so

$$
\frac{n_{c}}{l+1}=2 S_{\text {waste }}
$$

Adding eqns. (17) and (19), we get

$$
n_{0} s+2 S_{\text {waste }}=\frac{n_{c}+n_{t}}{l+1}=\frac{N-n_{b}-n_{0}}{l+1}
$$

or, rearranging the terms,

$n_{b}=\frac{N\left(1-2(l+1) f_{u} S_{\text {waste }}(\text { unocc })\right)}{2(l+1)\left(f_{c} S_{\text {waste }}(\text { occ })-f_{u} S_{\text {waste }}(\text { unocc })\right)+2}$

By plotting $n_{b}$ against $n_{0}$ using eqns. (16) and (21), we can obtain the equilibrium number of idle stations $n_{0 e}$ at the intersection of the two curves. The equilibrium throughput is then $S=n_{0 e} s$, and the total number of blocked or colliding stations at equilibrium is $n_{b e}+n_{c e}=N-n_{0 e}-S(l+1)$. Using Little's Formula, the delay at equilibrium is just $S /\left(n_{b e}+n_{c e}\right)$.

\section{RESULTS OF EPA AND COMPARISONS}

Table 1 shows the throughput and delay values for a CDMA-CS network, where $N=25$, $s=0.01, \quad l=30$ and where $p$ is varied, obtained from both EPA and simulation over 300,000 slots. We can see that the throughputs and delays predicted by EPA match those from simulations when the system is stable. For the $p=0.3$ case, the system is unstable, but occupied channels will only accumulate blocked stations, i.e., have $\Delta n_{k}>0$, when the number of blocked stations exceed 10. Simulation over 300,000 slots was not able to cause this to happen and force the system into the congested region. For the $p=0.5$ case, an occupied channel needs only 5 blocked stations before it will start accumulating more, and simulation over 300,000 slots was enough to see stations colliding at a common channel, and cause a tremendous drop in throughput, and a corresponding rise in message delays.

Table 2 shows the throughput and delay values for simple CSMA-CD and CDMA-CS/CD networks, as well as the CDMA-CS network, for various values of $s, p$ and $l$. All systems have $N=50$, and simulations for the CDMA-CS network are done over 100,000 slots. The throughputs and delays shown for the simple single-channel CSMA$\mathrm{CD}$ and the CDMA-CS/CD networks are obtained by EPA and taken from [1].

Comparing the throughputs and delays of the CDMA-CS network with those of the simple CSMA-CD and CDMA-CS/CD networks, we see that EPA predicts values for the CDMA-CS network that are only marginally worse than those of the CDMA-CS/CD network, and that are much better than those of the single channel CSMA-CD case. The throughput is practically the maximum possible, $N s$, and the message delays almost zero. Simulations over 100,000 slots for the multi-channel CSMA network was not able to yield any collisions, so the values obtained were much better than those predicted by EPA, and essentially the same as those obtained for the CDMA-CS/CD system. Note that the values of $s$ and $p$ are intentionally kept very low so that the simple CSMA-CD system does not enter the congestion region, and cause the throughput to drop so low that comparisons with the performances of the CDMA networks become meaningless.

\section{CONCLUSIONS}

Since the throughputs and delays offered by the CDMA-CS system are so much better than that of the simple single channel CSMA-CD network, and since a CDMA-CS network is much more easily implementable than a CDMA-CS/CD network, CDMA ALOHA with channel sensing is a form of multiple access that is worth implementing, especially in systems where stations are almost equally likely to transmit to one another, and where short message delays are essential. One added advantage of a CDMA radio network is that if it is lightly loaded, it can be placed in an environment where there are already existing narrow band radio users. The energy in a lightly loaded spreadspectrum channel will look just like weak wideband noise to those users, and pose little interference to them. 


\section{ACKNOWLEDGMENT}

This work was supported by the Hong Kong Research Grants Council and by the CRCG of The University of Hong Kong, Hong Kong.

\section{REFERENCES}

[1] F. L. Lo, T. S. Ng, T. I. Yuk, "Performance Analysis of A Fully-connected, Full-duplex CDMA ALOHA Network with Channel Sensing and Collision Detection", to appear in IEEE JSAC issue on CDMA Networks III, 1996

[2] W.C.Y. Lee, "Overview of CDMA", IEEE Trans. Veh. Technol, Vol. VTC-40, pp. 291-302, 1991

[3] S. Jiang, M. T. Hsiao, "Performance Evaluation of a Receiver-Based Handshake
Protocol for CDMA Networks", IEEE Trans. on Commun., Vol 43, pp. 2127 2138, 1995

[4] E. Sousa, J. Silvester, "Spreading Code Protocols for Distributed Spread-Spectrum Packet Radio Networks", IEEE Trans. on Commun., Vol. COM-36, pp. 272-281, 1988.

[5] K.-W. Yip, T.-S. Ng, "Code Phase Assignment - A Technique for High Capacity Indoor Mobile DS-CDMA Communications", IEEE 44th Veh. Tech. Conf., pp. 1586-1590, 1994

[6] L. Kleinrock, S. Lam, "Packet Switching in a Multiaccess Broadcast Channel: Performance Evaluation", IEEE Trans. on Commun., Vol. COM-23, pp. 410-423, 1975.

\begin{tabular}{|c|c|c|c|c|c|c|c|}
\hline \multirow{2}{*}{$s$} & \multirow{2}{*}{$p$} & \multicolumn{3}{|c|}{ EPA } & \multicolumn{2}{c|}{ Simulations } \\
\cline { 4 - 8 } & & & status & thruput & delay & thruput & delay \\
\hline \hline 0.01 & 0.10 & 30 & stable & 0.1669 & 18.79 & 0.1786 & 13.87 \\
\hline 0.01 & 0.15 & 30 & stable & 0.1687 & 17.19 & 0.1782 & 15.44 \\
\hline 0.01 & 0.20 & 30 & stable & 0.1696 & 16.41 & 0.1769 & 15.02 \\
\hline 0.01 & 0.30 & 30 & unstable & 0.1705 & 15.62 & 0.1753 & 16.56 \\
\hline 0.01 & 0.50 & 30 & unstable & 0.1713 & 14.95 & 0.0107 & 2217.8 \\
\hline
\end{tabular}

Table 1: Throughput and delay values for a CDMA-CS system with $N=25$, obtained from both EPA and simulation over 300,000 slots.

\begin{tabular}{|c|c|c|c|c|c|c|c|c|c|c|c|}
\hline \multirow[b]{3}{*}{$S$} & \multirow[b]{3}{*}{$p$} & \multirow[b]{3}{*}{$l$} & \multirow{2}{*}{\multicolumn{2}{|c|}{$\begin{array}{c}\text { Simple } \\
\text { CSMA-CD }\end{array}$}} & \multirow{2}{*}{\multicolumn{2}{|c|}{$\begin{array}{l}\text { CDMA- } \\
\text { CS/CD }\end{array}$}} & \multicolumn{5}{|c|}{ CDMA-CS } \\
\hline & & & & & & & \multicolumn{3}{|c|}{ EPA } & \multicolumn{2}{|c|}{ Simulat. } \\
\hline & & & t'put & delay & t'put & delay & status & t'put & delay & t'put & delay \\
\hline 0.001 & 0.05 & 10 & 0.048 & 26.9 & 0.050 & 0.40 & s'ble & 0.049 & 0.74 & 0.050 & 0.00 \\
\hline 0.001 & 0.05 & 20 & 0.041 & 213.6 & 0.049 & 1.02 & s'ble & 0.049 & 1.70 & 0.050 & 0.15 \\
\hline 0.001 & 0.10 & 10 & 0.049 & 13.2 & 0.050 & 0.20 & s'ble & 0.049 & 0.53 & 0.050 & 0.00 \\
\hline 0.001 & 0.10 & 20 & 0.042 & 181.1 & 0.049 & 0.61 & s'ble & 0.049 & 1.29 & 0.050 & 0.00 \\
\hline 0.002 & 0.05 & 10 & 0.073 & 186.5 & 0.098 & 0.71 & s'ble & 0.098 & 1.51 & 0.100 & 0.00 \\
\hline 0.002 & 0.05 & 20 & 0.042 & 697.8 & 0.096 & 1.77 & s'ble & 0.095 & 3.66 & 0.098 & 0.01 \\
\hline 0.002 & 0.10 & 10 & 0.072 & 194.2 & 0.098 & 0.51 & s'ble & 0.098 & 0.98 & 0.100 & 0.01 \\
\hline 0.002 & 0.10 & 20 & 0.033 & 1019 & 0.096 & 1.35 & s'ble & 0.095 & 2.67 & 0.098 & 0.01 \\
\hline 0.001 & 0.20 & 20 & \multicolumn{2}{|c|}{ unstable } & 0.049 & 0.61 & uns. & 0.049 & 1.08 & 0.050 & 0.00 \\
\hline 0.002 & 0.20 & 20 & \multicolumn{2}{|c|}{ congested } & 0.096 & 1.15 & uns & 0.096 & 2.23 & 0.098 & 0.01 \\
\hline
\end{tabular}

Table 2: Throughputs and delays for various values of $s, p$ and $l$, for the simple CSMA-CD, CDMA-CS/CD and CDMA-CS networks. $N=50$ and simulations are done over 100,000 slots. 